\title{
Limbic encephalitis - a report of four cases
}

\author{
ŻANNA PASTUSZAK ${ }^{1,2}$, ADAM STĘPIEN ${ }^{2}$, KAZIMIERZ TOMCZYKIEWICZ2, \\ RENATA PIUSIŃSKA-MACOCH', JOANNA KORDOWSKA ${ }^{3}$, DARIUSZ GALBARCZYK ${ }^{3}$, \\ JAROSEAW ŚWISTAK
}

${ }^{1}$ Department of Neurosurgery, Mossakowski Medical Research Centre, Polish Academy of Sciences, Warsaw, Poland

${ }^{2}$ Military Institute of Medicine, Warsaw, Poland

${ }^{3}$ Voivodeship Specialist Hospital, Ciechanow, Poland

\begin{abstract}
Usually limbic encephalitis (LE) is a paraneoplastic neurologic syndrome. LE symptoms can precede cancer even by a few years. Almost 50\% of LE cases are connected with small cell lung carcinoma. Testis and breast cancers, granulomatous disease, thymoma, and teratomas are also often connected with LE. Other cases have infectious and autoimmunological aetiology. In LE limbic system dysfunction is observed, and it is accompanied by cerebellum and brain stem abnormalities as well as polyneuropathy. Paraneoplastic limbic encephalitis is sometimes a part of larger syndrome in which brain stem and spinal cord are involved in an inflammatory process called paraneoplastic encephalomyelitis. The main LE symptoms are: impairment of cognitive functions with subacute beginning, partial and generalised seizures, mental distress, disturbances of consciousness, and limb paresis. In MRI study hyperintensive lesions in the medial part of the temporal lobes in T2 and FLAIR sequences are present. Sharp and slow waves in electroencephalography in the temporal area are also frequent. In cerebrospinal fluid pleocytosis, elevation of protein level, intensification of immunoglobulin synthesis, and oligoclonal bands can be detected. The majority of patients with paraneoplastic LE have onconeural antibodies in the blood. The presented study is a description of the clinical course of the disease in four patients diagnosed with LE.
\end{abstract}

Key words: limbic encephalitis, onconeural antibodies, paraneoplastic neurologic syndromes, tumour.

(Cent Eur J Immunol 2017; 42 (2): 213-217)

Limbic encephalitis was described for the first time in 1960 by Brievley and Corsellis. In LE limbic system dysfunction is observed, and it can be accompanied by cerebellum and brain stem abnormalities as well as polyneuropathy $[1,2]$. The disease pathogenesis remains elusive. The most popular theory says that the immunological response is mistakenly directed to limbic system antigens, which can be similar to those of cancer [3]. Almost 50\% of LE cases are connected with small cell lung carcinoma. Testis and breast cancers, granulomatous disease, thymoma, and teratomas are also frequently connected with LE [4]. Paraneoplastic limbic encephalitis is sometimes part of a larger syndrome in which brain stem and spinal cord are involved in the inflammatory process (paraneoplastic encephalomyelitis) [1, 2]. Limbic encephalitis can also have infectious or autoimmunological aetiology $[5,6]$.

\section{First case}

A 64-year-old patient with hypertension was admitted to the neurology department because of repetitive episodes of right limbs numbness, problems with speech, vertigo, and disturbances of consciousness, which had begun two weeks earlier. Initially performed neurological studies did not reveal abnormalities except sharp waves in the frontotemporal area of the brain in electroencephalography (EEG). The patient was diagnosed with epilepsy and was given carbamazepine in a dose of $2 \times 600 \mathrm{mg}$ with good result. In ambulation carbamazepine was changed to valproic acid in a dose of $2 \times 500 \mathrm{mg}$ because of hyponatraemia. Three years later the patient was admitted to hospital because of intensification of frequency of epilepsy attacks accompanied by deep vein thrombosis, dyspnoea, drowsiness during the day, psychosomatic slowness, and memory impairment. In Magnetic Resonance Imaging (MRI) study, lesions hyperintensive in T2 and hypointensive in $\mathrm{T} 1$ sequences, localised bilaterally in frontotemporal areas were observed. Laboratory tests revealed hyponatraemia, elevated level of C-reactive protein (CRP) and leucocytosis. In cerebrospinal fluid oligoclonal bands were detected and immunoglobulin synthesis was increased. Serological tests confirmed the presence of anti-Hu and anti-Yo antibodies in the patient's blood. In chest Computed Tomography $(\mathrm{CT})$ there was fluid in the pleural cavity and enlarged

Correspondence: Żanna Pastuszak, Military Institute of Medicine, Szaserow 128, 04-141 Warsaw, Poland, e-mail: zanna.pastuszak@ hotmail.com Submitted: 8.02.2016; Accepted: 18.05.2016 
lymph nodes in the mediastinum. During hospitalisation the patient was treated with immunoglobulin in a dose of $0.4 \mathrm{~g} / \mathrm{kg}$ for five days, but with no effect. After a few days, he died due to cardiac and respiratory insufficiency.

\section{Second case}

A 58-year-old woman was admitted to the neurology department because of behaviour disturbances and aphasia, which had begun a few days before hospitalisation. Laboratory tests revealed only leucocytosis. Neurological examination and brain TK study was normal. In MRI in the left temporal lobe hypointensive in $\mathrm{T} 1$ and hyperintensive in T2 sequences lesions were observed (Fig. 1). In cerebrospinal fluid oligoclonal bands, increased index and synthesis were detected. During hospitalisation deterioration of neurological status was observed. The patient had disturbances of consciousness, memory loss (especially short-term memory), and problems with concentration and orientation. In EEG, sharp waves in the temporal area were present. In the patient's blood anti-Hu and anti-Yo antibodies were present. During hospitalisation she was treated with Solu-Medrol in a dose of $1 \mathrm{~g} / 24 \mathrm{~h}$ for five days with no result. Oncological diagnostics did not reveal any abnormalities. The patient was discharged from hospital on demand.

\section{Third case}

A 71-year-old female was admitted to hospital because of psychomotor slowness, memory impairment, and deterioration of cognitive functions. Symptoms occurred three months before admission to the neurology department. Last year the patient was oncologically treated because of non-small cell lung cancer. Neurological examination revealed no abnormalities. Although MRI done three months

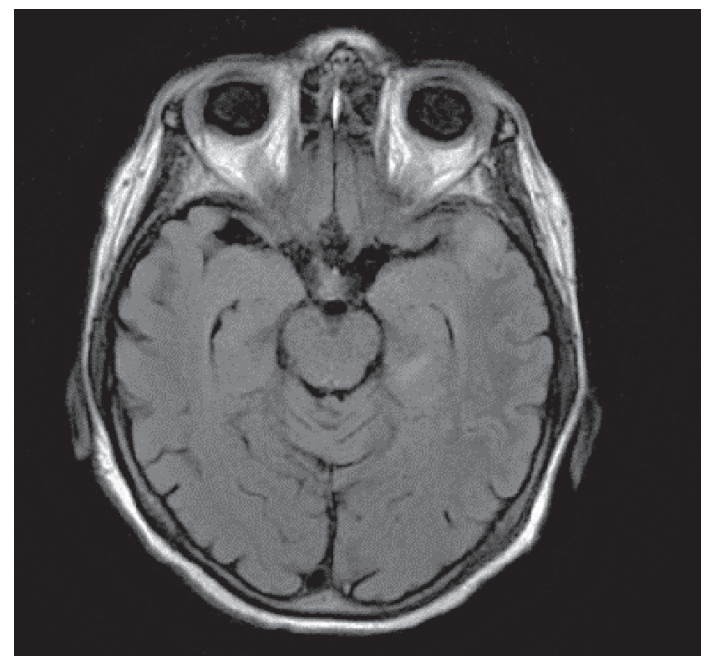

Fig. 1. Second patient MRI earlier was normal, the next examination revealed lesions hyperintensive in T2 and FLAIR sequences localised in temporal lobes (Fig. 2). Cerebrospinal fluid (CSF) index and synthesis were elevated, and oligoclonal bands were present. Leucocytosis and increased CRP were observed in the patient's blood. Sharp waves were seen in EEG in temporal areas. She was treated with Solu-Medrol in dose $2 \times 500 \mathrm{mg} / 24 \mathrm{~h}$ and valproic acid in dose $2 \times 500 \mathrm{mg} / 24$ $\mathrm{h}$ with no improvement. During hospitalisation the CRP value increased and the patient's condition deteriorated despite of treatment with vancomycin. Consequently, after a few days the patient died.

\section{Fourth case}

A 60-year-old female was admitted to the neurology department because of left facial nerve palsy with limb numbness, vertigo, and memory and orientation disturbances with psychomotor slowness. Symptoms had progressed for the preceding month. The patient suffered from ovarian cancer with metastases to the lungs. In neurological examination, there was sight impairment in the right eye, facial nerve palsy, and positive Romberg test. In MRI numerous lesions hyperintensive in $\mathrm{T} 2$ sequence localised in brain, cerebellum, and brain stem were observed (Fig. 3, 4). Some of these lesions were gadolinium enhanced. MRI revealed paraneoplastic lesions comorbid with angiogenic abnormalities and metastases. In CSF pleocytosis, elevated protein with decreased glucose level and oligoclonal bands were detected. In EEG there were sharp waves in temporal areas. In EMG study demyelinating polyneuropathy was diagnosed. Onconeural antibodies were absent from the

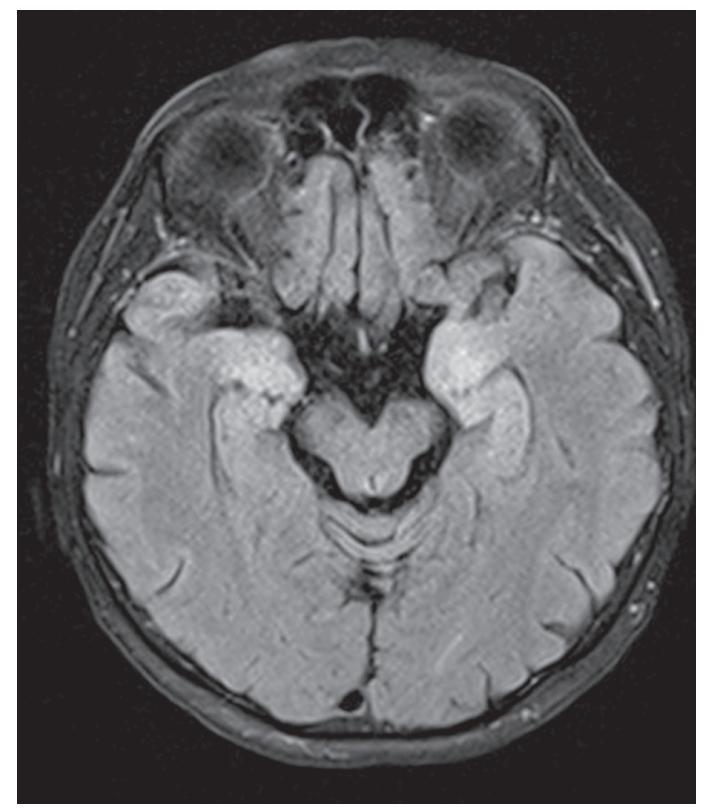

Fig. 2. Third patient's MRI 


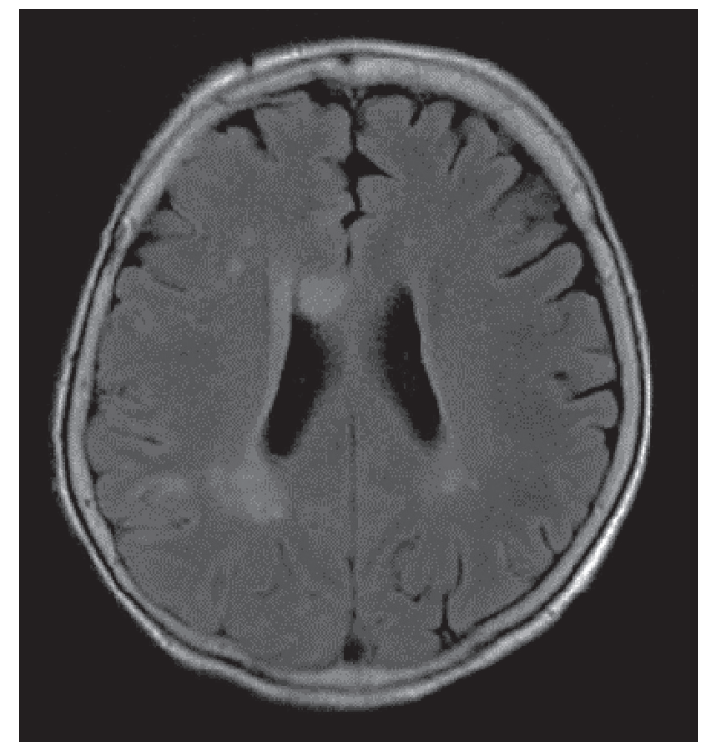

Fig. 3. Fourth patient's MRI

patient's blood. During hospitalisation the patient's neurologic condition deteriorated. Right limb paresis occurred and the patient died after a few days.

The most frequent symptoms of LE are cognitive performance impairment accompanied by epilepsy attacks and disturbances of consciousness. Patients often claim problems with memory and concentration. Psychiatric symptoms include anxiety, depression, and psychosis. A subacute beginning is characteristic for LE [7]. In the blood of patients with paraneoplastic LE anti-Hu, anti-Yo, anti-Ma2, anti-amphiphysin, and anti-CRMP5/CV2 antibodies can be detected $[5,6,8,9] .10-20 \%$ of patients with paraneoplastic LE are seronegative. The majority of them have lung cancer [10]. Anti-Hu antibodies are present in about half of LE patients [11]. LE can be connected with many oncological diseases. In the presented study two patients had diagnosis of lung carcinoma and one of breast and ovarian cancer. LE with VGKC antibodies (anti-voltage-gated potassium channel) is another subtype of disease connected with autoimmunological aetiology [12-14]. Other antibodies specific for non-paraneoplastic LE are anti-NMDAR (N-methyl D-aspartate receptor) and anti-nCMAg (novel cell membrane antigens) [15-18]. Benke proved that the antibody type is connected with patient prognosis. After eight months of observation $38 \%$ of patients with anti-Hu antibodies showed improvement of their neurological condition compared to $64 \%$ in the seronegative group [19]. Patients without onconeural antibodies and those with anty-Ma2 antibodies have better response to immunosuppressive treatment [20]. In the presented study, in the case of three patients, anti-Hu and anti-Yo antibodies were present, while a fourth patient was seronegative. Another LE subtype is called acute non-her-

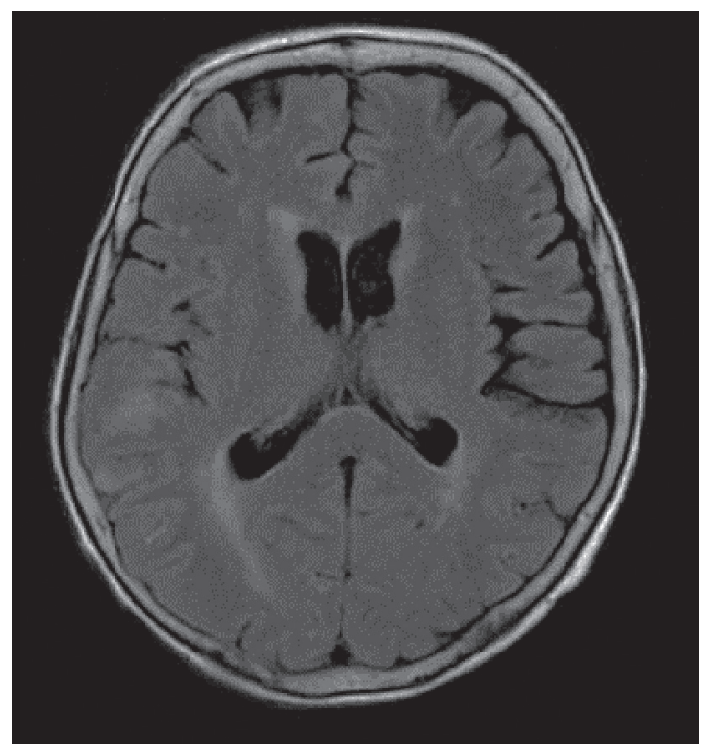

Fig. 4. Fourth patient's MRI

petic LE, and its clinical course and laboratory results are similar to those seen in central nervous system (CNS) infections. In those cases virological tests need to be done [21-23]. Hyponatraemia is the most frequently subtype observed in LE connected with VGKC antibodies [24]. In the first described case hyponatraemia may be a result of carbamazepine treatment.

Diagnostic criteria for LE are: characteristic result in histopathological examination, neuropsychiatric disorders, cancer diagnosed within a four year period, exclusion of symptoms connected with cancer, characteristic abnormalities in EEG and MRI, and inflammatory changes in cerebrospinal fluid (CSF) [25]. Some of these symptoms are common for all subtypes of LE. Acute or subacute cognitive performance impairment accompanied by epilepsy attacks are most frequently observed. The most common psychiatric disorders are: anxiety, psychosis, elevated mood, bipolar disorder, emotional lability, and behaviour disorders. In neurological examination, limb paresis and signs of brain stem and cerebellum involvement may be present as in the case of the fourth patient. All patients in the presented study had cognitive performance impairment, acute memory loss, depressive or elevated mood, anxiety, and emotional lability.

In MRI study lesions hyperintensive in T2 and FLAIR sequences localised in the medial part of the temporal lobes are observed $[9,26]$. They can be gadolinium-enhanced and typically are bilateral. In rare cases, MRI study reveal no abnormalities. In Lawn's study, abnormalities in MRI were observed in $82 \%$ of patients [27]. In the presented study, in all cases lesions were observed, and in the case of three patients they were localised bilaterally. In the case of the fourth woman, paraneoplastic abnormalities 
coexisted with metastases and vascular lesions localised in the brain stem and cerebellum. In the case of two patients, initially MRI did not reveal abnormalities in temporal lobes. In the first patient those changes were seen three years after the start of disease.

Oncological diagnostics are very important in patients with LE. In the first presented case that diagnosis was not completed because of the patient's death, and in the case of the second woman, it did not reveal cancer. Doctors should bear in mind that LE can precede neoplasms, even by few years, and should carefully monitor the patient's condition. Abnormalities in EEG are observed in 50-60\% of LE patients and include slow and sharp waves in temporal sites unilateral or bilateral [26, 27]. Lavn proved in his study that those abnormalities were similar in the case of seropositive and seronegative patients [28]. In the presented study, a 64-year-old man was initially diagnosed with epilepsy with simple partial seizures. Despite the fact that he was treated with carbamazepine, the attacks became more frequent and their morphology changed. Third patient did not have seizures but, due to the EEG result and cognitive function deterioration, was treated with valproic acid with no improvement.

CSF examination is also very important in the LE diagnostic process. The most frequently observed abnormalities are: lymphocytic pleocytosis, elevated protein level and immunoglobulin synthesis, and oligoclonal bands. In the described patients, all these changes were observed. A major role in the LE diagnostic process is played by neuropathological study. The most frequent LE features are: neuron loss in hippocampus and amygdala accompanied by perivascular lymphocytes infiltration and gliosis [29]. They are localised in the temporal lobe and in limbic system structures like the corpus mamillare, orbitofrontal cortex, and cingulate cortex [30]. In LE therapy it is necessary to remove cancer antigens with the use of surgery, radiotherapy, and chemotherapy. Also, immunosuppressive treatment such as immunoglobulins, steroids, or plasmapheresis is effective [31]. Improvement of neurological condition after treatment was rarely described in literature. In the presented study three patients died a few weeks after LE diagnosis. In the fourth case, immunosuppressive treatment was ineffective.

Limbic encephalitis is a rare disease with poor prognosis in the majority of patients. Further studies are needed to investigate the disease aetiology and to discover effective treatment.

\section{Conclusions}

Limbic encephalitis must be differed from inflammatory disease of CNS, especially with herpetic aetiology.

Patients with diagnosis of LE should undergo oncological diagnostics and observation.
MRI plays an important role in the diagnosis of LE and can reveal lesions hyperintensive in T2 and FLAIR sequences localised in the medial part of the temporal lobes.

For paraneoplastic LE the presence of anti-Hu, anti-Yo, anti-Ma2, anti-amphiphysin, and anti-CRMP5/CV2 antibodies is characteristic.

The authors declare no conflict of interest.

\section{References}

1. Brierley J, Corsellis J, Hierons R, et al. (1960): Subacute encephalitis of later adult life mainly affecting the limbic areas. Brain 83: 357-368.

2. Corsellis J, Goldberg G, Norton A (1968): "Limbic encephalitis" and its association with carcinoma. Brain 91: 481-496.

3. Dalmau J, Rosenfeld M (2008): Paraneoplastic syndromes of the CNS. Lancet Neurol 7: 327-340.

4. Gultekin S, Rosenfeld M, Voltz R, et al. (2000): Paraneoplastic limbic encephalitis: neurological symptoms, immunological findings and tumor association in 50 patients. Brain 123: 1481-1494.

5. Anderson N, Rosenblum M, Graus F, et al. (1988): Autoantibodies in paraneoplastic syndromes associated with small -cell lung cancer. Neurology 38: 1391-1398.

6. Dalmau J, Furneaux H, Rosenblum M, et al. (1991): Detection of the anti-Hu antibody in specific regions of the nervous system and tumor from patients with paraneoplastic encephalomyelitis/ sensory neuronopathy. Neurology 41: 1757-1764.

7. Posner J, Paraneoplastic syndromes. Neurologic complications of cancer, 1995;353-385.

8. Graus F, Cordon Cardo C, Posner J (1985): Neuronal antinuclear antibody in sensory neuronopathy from lung cancer. Neurology 35: 538-543.

9. Jubelt B, Cashman N (1987): Neurological manifestations of the post-polio syndrome. Crit Rev Neurobiol 3: 199-220.

10. Buckley C, Oger J, Clover L, et al. (2001): Potassium channel antibodies in two patients with reversible limbic encephalitis. Ann Neurol 50: 74-79.

11. Vincent A, Buckley C, Schott J, et al. (2004): Potassium channel antibody-associated encephalopathy: a potentially immunotherapy responsive form of limbic encephalitis. Brain 127: 701-712.

12. Thieben M, Lennon V, Boeve B, et al. (2004): Potentially reversible autoimmune limbic encephalitis with neuronal potassium channel antibody. Neurology 62: 1177-1182.

13. Saiz A, Blanco Y, Sabater L, et al. (2008): Spectrum of neurological syndromes associated with glutamic acid decarboxylase antibodies: diagnostic clues for this association. Brain 131: 2553-2563.

14. Mata S, Muscas G, Naldi I, et al. (2008): Non -paraneoplastic limbic encephalitis associated with anti- glutamic acid decarboxylase antibodies. J Neuroimmunol 199: 155-159.

15. Ances B, Vitaliani R, Taylor R, et al. (2005): Treatment-responsive limbic encephalitis identified by neuropil antibodies: MRI and PET correlates. Brain 128: 1764-1777.

16. Dalmau J., Tuzun E., Wu H, et al. (2007): Paraneoplastic anti -N -methyl -D -aspartate receptor encephalitis associated with ovarian teratoma. Ann Neurol 61: 25-36. 
17. Ramlow J, Alexander M, LaPorte R, et al. (1992): Epidemiology of the post-polio syndrome. Am J Epidemiol 136: 769-786.

18. Gołąb-Janowska M, Nowacki P (2011): Limbic encephalitis: a review of literature. Ann Acad Med Stet 57: 5-11.

19. Asaoka K, Shoji H., Nishizaka S, et al. (2004): Non herpetic acute limbic encephalitis: cerebrospinal fluid cytokines and magnetic resonance imaging findings. Intern Med 43: 42-48.

20. Bodian D (1947): Poliomyelitis: Neuropathologic observations in relation to motor symptoms. J Am Med Assoc 134: 1148-1154.

21. Zuliani L, Saiz A, Tavolato B, et al. (2007): Paraneoplastic limbic encephalitis associated with potassium channel antibodies: value of anti -glial nuclear antibodies in identifying the tumour. J Neurol Neurosurg Psychiatry 78: 204-205.

22. Tuzun E, Dalmau J (2007): Limbic encephalitis and variants: classification, diagnosis and treatment. Neurologist 13: 261271.

23. Daly D, Markand O (1990): Focal brain lesions. Curr Pract Clin Electroencephalography 335-370.

24. Lawn N, Westmoreland B, Kiely M, et al. (2003): Clinical, magnetic resonance imaging, and electroencephalographic findings in paraneoplastic limbic encephalitis. Mayo Clinic Proceedings 78: 1363-1368.

25. Nowacki P (2008): Neuropatologic basis of paraneoplastic syndromes. Pol Rev Neurol 4: 168-174.

26. Brierley J, Corsellis J, Hierons R, et al. (1960): Subacute encephalitis of later adult life, mainly affecting the limbic areas. Brain 83: 357-368.

27. Alamowitch, F, Graus M, Uchuya R, et al. (1997): Limbic encephalitis and small cell lung cancer. Clinical and immunological features. Brain 120: 923-928. 